Article

\title{
Observability and Symmetries of Linear Control Systems
}

\author{
Víctor Ayala ${ }^{1, *}$, Heriberto Román-Flores ${ }^{1}$, María Torreblanca Todco ${ }^{2}$ and Erika Zapana ${ }^{2}$ \\ 1 Instituto de Alta Investigación, Universidad de Tarapacá, Casilla 7D, Arica 1000000, Chile; hroman@uta.cl \\ 2 Departamento Académico de Matemáticas, Universidad, Nacional de San Agustín de Arequipa, Calle Santa \\ Catalina, Nro. 117, Arequipa 04000, Peru; mtorreblancat@unsa.edu.pe (M.T.T.); ezapana@unsa.edu.pe (E.Z.) \\ * Correspondence: vayala@uta.cl
}

Received: 7 April 2020; Accepted: 6 May 2020; Published: 4 June 2020

check for updates

\begin{abstract}
The goal of this article is to compare the observability properties of the class of linear control systems in two different manifolds: on the Euclidean space $\mathbb{R}^{n}$ and, in a more general setup, on a connected Lie group G. For that, we establish well-known results. The symmetries involved in this theory allow characterizing the observability property on Euclidean spaces and the local observability property on Lie groups.
\end{abstract}

Keywords: observability; symmetries; Euclidean spaces; Lie groups

\section{Introduction}

For general facts about control theory, we suggest the references, [1-5], and for general mathematical issues, the references [6-8].

In the context of this article, a general control system $\Sigma$ can be modeled by the data $\Sigma=(M, D, h, N)$. Here, $M$ is the manifold of the space states, $D$ is a family of differential equations on $M$, or if you wish, a family of vector fields on the manifold, $h: M \rightarrow N$ is a differentiable observation map, and $N$ is a manifold that contains all the known information that you can see of the system through $h$. Normally, $\operatorname{dim}(N) \leq \operatorname{dim}(M)$. One of the main problems here is to find topological, algebraic, and differentiable properties to impose on $\Sigma$, to recover the dynamics on $M$, through the partial information given by $h$.

The indistinguishable equivalence relation associated with the data permit decomposing the manifold into subsets where two points in the same set can not be distinguishable for $D$ and $h$ in $N$. More precisely, given two distinguishable states $x, y \in M$, there exist positive times $t_{1}, \ldots, t_{l}>0$ with $t=t_{1}+\ldots+t_{l}$ and a concatenation $\varphi_{t}=\varphi_{t_{1}} \circ \ldots \circ \varphi_{t_{l}}$ of flows associated with vector fields $X^{1}, \ldots, X^{l} \in D$ such that:

$$
h\left(\varphi_{t}(x)\right) \neq h\left(\varphi_{t}(y)\right) .
$$

The system is said to be observable if any indistinguishable class is a singleton set. In other words, any two points of $M$ are distinguishable by $\Sigma$.

The goal of this article is just to compare the observability properties of the class of linear control systems in two different manifolds: on the Euclidean space $\mathbb{R}^{n}$ and, in a more general setup, on a connected Lie group $G$. The symmetries involved in this theory allow:

1. characterizing the observability property on $\mathbb{R}^{n}$ and also

2. characterizing the local observability property on $G$. After that, it is possible to get the global observability property on $G$ through the fixed points of the drift vector field of the system.

In order to compare both systems, we establish well-known observability results in both cases. We cite the corresponding references. 
Linear control systems on $\mathbb{R}^{n}$ appear in many applications [9-18] for a related control system. Markus in [12] extended this notion from $\mathbb{R}^{n}$ to a matrix group. Finally, in [19], the authors introduced the class of linear control system on any Lie group. In the references [18-35], the authors addressed relevant problems for this class of systems.

Furthermore, in [26], the author showed that each affine control system that generates a finite-dimensional Lie algebra on a connected manifold is diffeomorphic to a linear control system on a Lie group, or on a homogeneous space. This result shows the relevance of this class of systems on applications.

The article is organized as follows. In Section 2, we recall the notion of linear control systems $\Sigma_{\mathbb{R}^{n}}$ on Euclidean spaces. We establish the main observability results for $\Sigma_{\mathbb{R}^{n}}$ and the special symmetry contained in the data of the system, which completely determine this property. We finish the section with a couple of concrete examples. One of them is motivated by an example appearing in the book by Pontryagin [4]. Section 3 introduces the linear control system on connected Lie groups $G$. We show the face of the solution of this system and the notion of local observability and the (global) observability, and its main results appear in $[27,28]$. Before giving examples, we establish an algorithm to compute the relevant algebraic tools to decide the observability for the linear control system with observation $\Sigma_{G}^{\mathcal{O}}$. We finish the section with a couple of examples. Finally, in the Appendix A, we give a short review on Lie theory to make the article more self-contained, [29].

\section{Linear Control Systems on Euclidean Spaces}

To introduce the definition of the linear control system on Euclidean spaces in this first section, we introduce elementary dynamic system notions about two classes of dynamics: linear and invariant.

Let us denote by $\mathbb{R}$ the set of real numbers. The Euclidean space $\mathbb{R}^{n}=\left\{x=\left(x_{1}, \ldots, x_{n}\right): x_{i} \in \mathbb{R}\right\}$ is generated by the basis vectors:

$$
e_{i}=(0, \ldots, 1, \ldots, 0)=\left(\frac{\partial}{\partial x_{i}}\right)_{0}, i=1, \ldots, n .
$$

As an analytical differentiable manifold, the tangent space $T_{x} \mathbb{R}^{n}$ of $\mathbb{R}^{n}$ at the state $x \in \mathbb{R}^{n}$ is given by the vector space generated by:

$$
\left(\frac{\partial}{\partial x_{i}}\right)_{x}: i=1, \ldots, n,
$$

where the vector $\left(\frac{\partial}{\partial x_{i}}\right)_{x}$ is obtained by the translation of $e_{i}$ by $x$.

A linear control system on $\mathbb{R}^{n}$ essentially controls the behavior of a linear differential equation (linear vector field) through a number of constant vector fields, i.e., invariant (by translation) vector fields on $\mathbb{R}^{n}$.

In the sequel, we consider $\mathfrak{g l}(n, \mathbb{R})$ the vector space of real matrices of order $n$. Any element $A \in \mathfrak{g l}(n, \mathbb{R})$ determines a linear differential equation $\dot{x}(t)=A x(t)$ with $x(0)=x_{0}$ as the initial condition. The corresponding solution reads as $x(t)=e^{t A} x_{0}$, where $e^{A}=\sum_{j=0}^{\infty} A_{j !}^{j}$ and $A^{0}=I d$ is the identity map on $\mathbb{R}^{n}$. Therefore, $A$ induces the linear vector field:

$$
x \stackrel{A}{\longrightarrow} \dot{x}(t)=A x(t) \in T_{x(t)} \mathbb{R}^{n}
$$

Then, the flow of the linear vector field $X^{A}$ defined by $A$ reads as:

$$
X_{t}^{A}: \mathbb{R}^{n} \rightarrow \mathbb{R}^{n}, X_{t}^{A}(x)=e^{t A} x, t \in \mathbb{R} .
$$

On the other hand, any vector $b=\left(b_{1}, \ldots, b_{i}, \ldots, b_{n}\right) \in \mathbb{R}^{n}$ defines an invariant vector field $X^{b}$ on $\mathbb{R}^{n}$ by:

$$
X^{b}(x)=\sum_{i=0}^{n} b_{i}\left(\frac{\partial}{\partial x_{i}}\right)_{x} \in T_{x} \mathbb{R}^{n}
$$


the solution of which is determined by its flows $X_{t}^{b}(x)=x+t b$, parallel lines to the line generated by $b$.

A linear control system on the Euclidean space $\mathbb{R}^{n}$ is defined by the family of ordinary differential equations:

$$
\Sigma_{\mathbb{R}^{n}}: \dot{x}(t)=A x(t)+B u, u \in \mathcal{U},
$$

parametrized by the controls $u \in \mathcal{U}$. Here, $x \in \mathbb{R}^{n}, A \in \mathfrak{g l}(n, \mathbb{R})$ is a square real matrix of order $n, B$ is an $n \times m$ real matrix, and $\mathcal{U}=L_{\text {loc }}^{1}(\mathbb{R}, \Omega)$ is the family of admissible control functions. The set $\Omega \subset \mathbb{R}^{m}$ is closed, and $0 \in \operatorname{int}(\Omega)$. In other words, $\mathcal{U}$ is the space of locally integrable measure functions $u: \mathbb{R} \rightarrow \Omega$.

Consider a state $x_{0} \in \mathbb{R}^{n}$ and a control $u \in \mathcal{U}$. The associated solution of $\Sigma_{\mathbb{R}^{n}}$ with these two parameters reads as:

$$
\varphi_{t}^{u}\left(x_{0}\right)=e^{t A}\left(x_{0}+\int_{0}^{t} e^{-\tau A} B u(\tau) d \tau\right) .
$$

In fact, $\varphi_{t}^{u}\left(x_{0}\right)$ is the only absolutely continuous function satisfying the Cauchy problem: [30]

$$
\varphi_{t}^{u}\left(x_{0}\right)=A \varphi_{t}^{u}\left(x_{0}\right)+B u, \quad \varphi_{0}^{u}\left(x_{0}\right)=x_{0},
$$

The solution set $\left\{\varphi_{t}^{u}\left(x_{0}\right): t \in \mathbb{R}\right\}$ describes a curve in $\mathbb{R}^{n}$ in which elements are reached by $x_{0}$ through the specific dynamics $u$ of $\Sigma_{\mathbb{R}^{n}}$ in positive and negative time. More generally, the positive orbit of $\Sigma_{\mathbb{R}^{n}}$ starting on any initial condition $x_{0}$ is given by:

$$
S\left(x_{0}\right)=\left\{\varphi_{t}^{u}\left(x_{0}\right): 0 \leq t, u \in \mathcal{U}\right\} .
$$

Thus, $S\left(x_{0}\right)$ is the set of the states of $\mathbb{R}^{n}$ that can be reached from $x_{0}$ in positive time by using all the control functions $u \in \mathcal{U}$.

\subsection{Observability}

A linear control system with observation $\Sigma_{\mathbb{R}^{n}}^{\mathcal{O}}$ is a linear control system on $\mathbb{R}^{n}$ given by the data contained in Formula (1) and the observation map $h: \mathbb{R}^{n} \rightarrow \mathbb{R}^{s}$. Here, $s \leq n$ and $h$ is a linear transformation $[30,31]$.

Our interest is in analyzing the observability property of $\Sigma_{\mathbb{R}^{n}}^{\mathcal{O}}$ and a special symmetry property of the pair $(A, h)$ determining this notion. All the results in this section are very well known [30]. However, we need to include them in order to compare with the Lie group case.

In the sequel, we introduce the $\Sigma_{\mathbb{R}^{n}}^{\mathcal{O}}$-indistinguishable relationship in $\mathbb{R}^{n}$. This equivalence relation allows us to decompose the space of states into equivalence classes, which contain those elements that cannot be distinguished from each other in $\mathbb{R}^{s}$ by the observation map $h: \mathbb{R}^{n} \rightarrow \mathbb{R}^{s}$ through every positive dynamic of $\Sigma_{\mathbb{R}^{n}}^{\mathcal{O}}$. Precisely, we denote by $C$ the matrix of $h$ in the canonical basis. The linear map $h$ does not distinguish the state $x_{1}$ from $x_{0}$ through any positive dynamic of $\Sigma_{\mathbb{R}^{n}}^{\mathcal{O}}$, meaning that for any control $u \in \mathcal{U}$ and for each time $t \geq 0$ :

$$
C \varphi_{t}^{u}\left(x_{1}\right)=C \varphi_{t}^{u}\left(x_{0}\right) .
$$

This condition is equivalent to:

$$
\begin{gathered}
C\left(e^{t A}\left(x_{0}+\int_{0}^{t} e^{-\tau A} B u(\tau) d \tau\right)\right)=C\left(e^{t A}\left(x_{1}+\int_{0}^{t} e^{-\tau A} B u(\tau) d \tau\right)\right) \Leftrightarrow \\
C e^{t A} x_{0}=C e^{t A} x_{1} \text {, for each } t \geq 0
\end{gathered}
$$


in particular, when $t=0, C x_{0}=C x_{1}$.i Recall that the kernel and the image of $h$, given respectively by:

$$
\operatorname{Ker}(h)=\left\{x \in \mathbb{R}^{n}: h(x)=0\right\}, \operatorname{Im}(h)=\left\{y \in \mathbb{R}^{s}: \exists x \in \mathbb{R}^{n} \text { with } y=h(x)\right\}
$$

are subspaces of $\mathbb{R}^{n}$.

Definition 1. Let $\Sigma_{\mathbb{R}^{n}}^{\mathcal{O}}$ be a linear control system with observation:

1. Two states $x_{0}, x_{1} \in \mathbb{R}^{n}$ are indistinguishable for $\Sigma_{\mathbb{R}^{n}}^{\mathcal{O}}$, denoted as $x_{0} I x_{1}$, if:

$$
x_{1}-x_{0} \in \operatorname{Ker}\left(C e^{t A}\right), \forall t \geq 0
$$

2. $\Sigma_{\mathbb{R}^{n}}^{\mathcal{O}}$ is said to be observable from $x \in \mathbb{R}^{n}$ if the equivalence class $I(x)$ of $x$ is $\{x\}$.

3. $\Sigma_{\mathbb{R}^{n}}^{O}$ is said to be observable if it is observable from $x$ for each $x \in \mathbb{R}^{n}$.

The main results of this section show that the observability property of linear control systems with observation totally depends on a symmetric matrix built with $A$ and $C$. The next result shows some very well-known properties of $I(0)$, which determine completely the observability property of the system; see [30,31]. Since the proof is simple, we include it because it will inspire obtaining symmetric properties on the Lie group case.

Proposition 1. Let $\Sigma_{\mathbb{R}^{n}}^{\mathcal{O}}$ be a linear control system with observation. Then,

1. I is an equivalence relation;

2. $I(0)=\underset{t \geq 0}{\cap} \operatorname{Ker}\left(C e^{t A}\right)$;

3. $I(x)=x+I(0)$.

Proof. To prove the first item, we use the fact that for any $t \geq 0$, the set $\operatorname{Ker}\left(C e^{t A}\right) \subset \mathbb{R}^{n}$ is a vector subspace. It follows that $x I x$ and also $x I y$ imply $y I x$. Thus, $I$ is reflexive and symmetric. Now, assume $x_{0} I x_{1}$ and $x_{1} I x_{2}$. The equality:

$$
x_{2}-x_{0}=\left(x_{1}-x_{0}\right)+\left(x_{2}-x_{1}\right) \in \operatorname{Ker}\left(C\left(e^{t A}\right)\right), \forall t \geq 0
$$

shows that $I$ is also transitive.

To prove 2, consider $x \in I(0)$. By definition, the analytical curve $\gamma(t)=C\left(e^{t A}\right) x=0$ for any $t \geq 0$. Taking the first derivative of $\gamma$, we obtain:

$$
\left(\frac{d}{d t}\right)_{t} C\left(e^{t A}\right) x=C A\left(e^{t A}\right) x=0 .
$$

When $t=0$, we get $x \in \operatorname{Ker}(C A)$. By taking the derivative of $C A\left(e^{t A}\right) x=0$ at $t=0$, we obtain $C A^{2} x=0$. Continuing this process, we obtain:

$$
I(0) \subset \bigcap_{j=0}^{n-1} \operatorname{Ker}\left(C A^{j}\right) .
$$

On the other hand, if $x \in \bigcap_{j=0}^{n-1} \operatorname{Ker}\left(C A^{j}\right)$, it follows immediately that:

$$
x \in \operatorname{Ker}\left(C e^{t A}\right), \forall t \geq 0
$$

In fact, from the Cayley-Hamilton theorem [30], $A^{l} \in \operatorname{Span}\left\{A^{1}, A^{2}, \ldots, A^{n-1}\right\}$ for $l \geq n$. Since, $\operatorname{Ker}\left(C A^{j}\right) x=0$, for $j=1, \ldots, n-1$, the conclusion follows from the definition of the exponential map $e^{t A}=\sum_{j=0}^{\infty} t^{j} A^{j}$ and $A^{0}=I d$. 
Finally, let $x \in \mathbb{R}^{n}$ be arbitrary by fixing $y \in I(x)$. By definition:

$$
y-x \in \operatorname{Ker}\left(C\left(e^{t A}\right)\right), \forall t \geq 0 .
$$

Thus,

$$
y-x \in I(0) \Leftrightarrow y \in x+I(0) \Rightarrow I(x) \subset x+I(0) .
$$

Let $y \in I(0)$. Since, $y=(x+y)-x \in I(0)$; then $(x+y)+I x$; therefore, $x+y \in I(x)$, concluding the proof.

From the previous proposition, any indistinguishable equivalence class $I(x)$ depends just on $x$ and $I(0)$. Therefore, we get the main results of this section:

Theorem 1. Let $\Sigma_{\mathbb{R}^{n}}^{\mathcal{O}}$ be a linear control system with observation:

$$
\Sigma_{\mathbb{R}^{n}}^{\mathcal{O}} \text { is observable } \Leftrightarrow I(0)=\{0\} .
$$

\subsection{The Symmetric Matrix Determining Observability on $\mathbb{R}^{n}$}

Next, we show that the observability property of a linear system with observation depends strongly on the symmetric Gramian observability matrix, [30]

$$
G_{0}(T)=\int_{0}^{T} e^{\tau A^{*}} C^{*} C e^{\tau A} d \tau,
$$

where $P^{*}$ denotes the transpose of the matrix $P$.

Theorem 2. Let $\Sigma_{\mathbb{R}^{n}}^{\mathcal{O}}$ be a linear control system with observation. Therefore, for any $T>0$ :

$$
I(0)=\operatorname{Ker} G_{0}(T) .
$$

Proof. The proof is independent of $T$. If $x \in \operatorname{Ker} G_{0}$, hence $x^{*} G_{0} x=0$. Thus,

$$
x^{*}\left(\int_{0}^{T} e^{\tau A^{*}} C^{*} C e^{\tau A} d \tau\right) x=\int_{0}^{T}\left\|C e^{\tau A} x\right\|^{2} d \tau=0 .
$$

Since the application $C e^{\tau A} x:[0, T] \rightarrow \mathbb{R}^{n}$ is analytical, the continuous function $\left\|C e^{\tau A} x\right\|^{2}$ must be null on the interval $[0, T]$. In fact, if there exists $\tau$ with $\left\|C e^{\tau A} x\right\|^{2}>0$, by continuity, there exits $\epsilon>0$ and a neighborhood $(\tau-\varepsilon, \tau+\varepsilon)$ where $\left\|C e^{t A} x\right\|^{2}>0$. Hence,

$$
\int_{0}^{T}\left\|C e^{\tau A} x\right\|^{2} d \tau>0
$$

and we have a contradiction. Therefore,

$$
\gamma(\tau)=C e^{\tau A} x=0,0 \leq \tau \leq T .
$$

However, $\gamma$ is an analytical application, which vanishes on the open set $(0, T)$. Therefore, $\gamma$ must be null on the whole real line. In particular, $C e^{t A} x=0$, for each $t \in \mathbb{R}$. As before, we obtain $x \in I(0)$.

Reciprocally, if $x$ is indistinguishable from the origin,

$$
G_{0} x=\int_{0}^{T} e^{\tau A^{*}} C^{*} C e^{\tau A} d \tau=\int_{0}^{T} e^{\tau A^{*}} C^{*} 0 d \tau=0,
$$


and the proof is complete.

Remark 1. Since for any $T>0$, the matrix $G_{0}(T)$ is symmetric, it is possible to consider its orthogonal space:

$$
\operatorname{Ker} G_{0}(T)^{\perp}=I_{m} G_{0}(T) .
$$

In particular, $I_{m} G_{0}(T)$ is the space of the observable states of the system. On the other hand, any $x \in \mathbb{R}^{n}$ has a unique decomposition:

$$
x=x_{0}+x_{I}, \quad x_{0} \perp x_{I}
$$

where $x_{0}$ is the observable component of $x$ and $x_{I}$ is the non-observable part.

To analyze observability through the Gramian matrix, you need to compute an integral. However, it is possible to avoid this calculus through the following algebraic characterization.

Theorem 3. Let $\Sigma_{\mathbb{R}^{n}}^{\mathcal{O}}$ be a linear control system with observation. Denote by $\mathcal{O} \in M_{s n \times n}(\mathbb{R})$ the matrix:

$$
\mathcal{O}=\left(C C A C A^{2} \ldots C A^{n-1}\right)^{\text {Transposed }} .
$$

Then, $I(0)=\operatorname{Ker}(\mathcal{O})$.

Proof. The proof follows directly from:

$$
x \in I(0) \Leftrightarrow C A^{j} x=0, j=0,1, . ., n-1 \Leftrightarrow C e^{t A} x=0, \forall t \geq 0 .
$$

As a consequence of the previous analysis, we obtain the following directly:

Corollary 1. Let $\Sigma_{\mathbb{R}^{n}}^{\mathcal{O}}$ be a linear control system with observation and $T>0$. The following are equivalent:

1. $\Sigma_{\mathbb{R}^{n}}^{\mathcal{O}}$ is observable;

2. $G_{0}(T)$ is invertible for any $T>0$;

3. $\operatorname{Rank}(\mathcal{O})=n$.

\subsection{Examples}

The first example shows that it is possible to build trajectories through the observation function when the system is observable. This means, in this case, that it is possible to determine the solutions of the system on $\mathbb{R}^{n}$ just with $s<n$ parameters.

Example 1. From an observation function, recover the optimal trajectories of the following problem:

\section{Stopping a train at the railway station in the shortest time.}

This very famous optimal problem appears in the book [4]. We extend this model with a couple of observation maps.

Let us consider a railway modeled by the real line and without friction. We denote by $x(t)$ the distance between the train and the origin $0 \in \mathbb{R}$ considered as the station. Then, we get on $\mathbb{R}^{2}$ the linear control system:

$$
\Sigma_{\mathbb{R}^{2}}:\left(\begin{array}{c}
\dot{x}(t) \\
\dot{y}(t)
\end{array}\right)=\left(\begin{array}{cc}
0 & 1 \\
0 & 0
\end{array}\right)\left(\begin{array}{c}
x(t) \\
y(t)
\end{array}\right)+\left(\begin{array}{l}
0 \\
1
\end{array}\right) u(t), u \in \mathcal{U} .
$$


In fact, from Newton's law, $F=M u$, where the function $u$ is the acceleration. Of course, we can consider $M=1$, then we get:

$$
\dot{x}(t)=y(t) \text { is the velocity and } \dot{y}(t)=u(t) \text { the acceleration. }
$$

The train is controlled by the admissible class of control $\mathcal{U}=L_{l o c}^{1}(\mathbb{R}, \Omega=[-1,1])$, and the set

$$
\mathcal{U}=\{u: \mathbb{R} \rightarrow \Omega, u \text { is locally integrable }\} .
$$

Here, $-1 \leq u(t) \leq 1$ with 1 and -1 representing the maximum and minimum possible accelerations and breaking, respectively. It is interesting to observe the geometric meaning of the problem: to solve it, we need first to associate with any initial state $z_{0}=\left(x_{0}, y_{0}\right) \in \mathbb{R}^{2}$ a curve solution of the system $\Sigma_{\mathbb{R}^{2}}$ transporting $z_{0}$ to the origin $(0,0)$.

$\operatorname{Rank}(\mathcal{O})=\left(\begin{array}{ll}0 & 1 \\ 1 & 0\end{array}\right)=2$. On the other hand, the Lyapunov spectrum $\operatorname{Spec}_{L y}(A)$ of $A$, built with the real parts of the eigenvalues of $A$ is equal to $\{0\}$. According to Example 3.2.16 in the book of Colonius and Kliemann [1], the system is controllable, which means that given any two points of the plane, each of them can be reached from the other through a solution of the system in positive time. In particular, the optimal problem is well posed. Hence, from a trivial computation, we know that for any state $z$, there exists $u \in \mathcal{U}$ and a +time $t>0$ such that $\varphi_{0}^{u}\left(z_{0}\right)=z_{0}$ and $\varphi_{t}^{u}\left(z_{0}\right)=$ $(0,0)$. However, we do not know what is the curve that does the job. Worst, among all the curves transporting $z_{0}$ to the origin, how doe we compute the trajectory with the shortest time? Fortunately, by the Pontryagin maximum principle [4], the optimal control $u^{*}$ exists, and its optimal value belongs to the boundary $\partial \Omega=\{-1,1\}$. Furthermore, according to this principle, the optimal trajectories are concatenations with at most one change (i.e., the dimension of the manifold $n$ minus one), of two main curves: the solutions of $u=-1$ and $u=1$. Each of the differential equations induced by these special controls decomposes the plane into (parallel) parabolas with respect to the $y$-axis, solving the problem completely. It is worth saying that this principle [4] won a relevant prize in Russia. Just observe that you reduce the possible values of the optimal trajectory from the interval $[-1,1]$ to the discrete set $\{-1,1\}$; from non-numerable infinity values to two possibilities.

Now, we apply the observability results to show that for the train problem, it is more relevant to know the first variable rather than the second one, i.e., knowing the distances gives you more information than the velocities.

1. Consider $\Sigma_{\mathbb{R}^{2}}$ and $h=\pi_{1}: \mathbb{R}^{2} \rightarrow \mathbb{R}, h(x, y)=x$ the projection on the first variable. A simple computation shows that $\operatorname{Rank}(\mathcal{O})=2$. In fact,

$$
C=\left(\begin{array}{ll}
1 & 0
\end{array}\right), A=\left(\begin{array}{ll}
0 & 1 \\
0 & 0
\end{array}\right), C A=\left(\begin{array}{ll}
0 & 1
\end{array}\right) \text { then } \mathcal{O}=\left(\begin{array}{ll}
1 & 0 \\
0 & 1
\end{array}\right) \text {. }
$$

According to Theorem 3, the system is observable.

2. Consider now the same system $\Sigma_{\mathbb{R}^{2}}$ and $h=\pi_{2}: \mathbb{R}^{2} \rightarrow \mathbb{R}$ with $h(x, y)=y$ the projection on the second variable. In this case:

$$
C=\left(\begin{array}{ll}
0 & 1
\end{array}\right), C A=\left(\begin{array}{ll}
0 & 0
\end{array}\right) \text { then } \mathcal{O}=\left(\begin{array}{ll}
0 & 0 \\
1 & 0
\end{array}\right) .
$$

and $\operatorname{Rank}(\mathcal{O})=1$. Therefore, the system is not observable. Furthermore, $I(0,0)$ coincides with the $x$-axis. For any $z \in \mathbb{R}^{2}, I(z)=z+\mathbb{R}$.

Geometrically, this situation can be explained as follows. In the case of $\pi_{1}$, any two states with different first coordinates can be distinguished directly from their own observable function. 
Therefore, take any two states with the first coordinate equal to zero. By applying simultaneously the solutions coming from $u=1$ to these two initial conditions, the projection of both curves will coincide. Now, by considering $u=-1$, the projections of the curves will give different trajectories on the $x$-axis. Therefore, the system is observable since $I(0)=\{0\}$.

In the case of $\pi_{2}$, consider two different states on the $x$-axis. The projections to the $y$-axis of the mentioned parabolas with $u=1$ and $u=-1$, will coincide. Therefore, $I(0)=x$-axis, and the system is not observable. In particular, through the knowledge of the velocities, it is not possible to recover the solution in the manifold. More precisely, contrary to the first case, here we cannot determine the initial condition and therefore all the trajectory!

Example 2. The next example corresponds to a linearization of a non-linear control system modeling the attitude of a satellite in orbit around the Earth (see [2]) with different observation maps.

Let $\Sigma_{\mathbb{R}^{n}}$ be a linear control system with:

$$
A=\left(\begin{array}{cccc}
0 & 1 & 0 & 0 \\
3 \beta^{2} & 0 & 0 & 2 \beta \\
0 & 0 & 0 & 1 \\
0 & -2 \beta & 0 & 0
\end{array}\right), B=\left(\begin{array}{cc}
0 & 0 \\
1 & 0 \\
0 & 0 \\
0 & 1
\end{array}\right), \beta \neq 0
$$

1. Consider the linear transformation $h$ with matrix $C=\left(\begin{array}{llll}1 & 0 & 0 & 0 \\ 0 & 0 & 1 & 0\end{array}\right)$. A simple computation shows that $\mathcal{O}$ is invertible. Hence, the system is observable.

2. If $C=\left(\begin{array}{llll}1 & 0 & 0 & 0\end{array}\right): \mathbb{R}^{4} \rightarrow \mathbb{R}, \operatorname{Rank}(\mathcal{O})=3$, and the system is not observable. Therefore, by knowing just the first variable, it is not possible to rebuild the system.

3. If $C=\left(\begin{array}{llll}0 & 0 & 1 & 0\end{array}\right): \mathbb{R}^{4} \rightarrow \mathbb{R}, \operatorname{Rank}(\mathcal{O})=4$, and the system is observable. Hence, the third variable contains enough information to recover any $\Sigma$-trajectories from a given initial condition.

\section{Linear Control Systems on Lie Groups}

The Appendix at the end of the article contains a short review of Lie theory; mainly, a basic notion about Lie groups, Lie algebras, and some examples. The reference [29] is a very good starting point.

We begin this section by extending the definition of a linear control system from the Euclidean space $G=\mathbb{R}^{n}$ to a connected arbitrary Lie group $G$. From a dynamic point of view, a linear control system on the Abelian group $\mathbb{R}^{n}$ is determined by:

$$
\text { the } \operatorname{drift} A \in \mathfrak{g l}(n, \mathbb{R}) \text {, and the } B \text {-column vectors } b^{j} \in \mathbb{R}^{n}, j=1, \ldots, m \text {. }
$$

We start by the drift vector field $A$. For that, we need to extend the notion of the linear differential equation $\dot{x}(t)=A x(t)$ from vector spaces to groups.

The first idea comes from the flow $e^{t A}: \mathbb{R}^{n} \rightarrow \mathbb{R}^{n}, t \in \mathbb{R}$, induced by matrix $A$, which is a one-parameter group of $\mathbb{R}^{n}$-automorphism. In fact, for any real number $t$, the linear map $e^{-t A}$ is the inverse of $e^{t A}$. As a first approach, we impose on $\mathcal{X}$ the same property. This means that the flow $\varphi_{t}$ of the drift $\mathcal{X}$ :

$$
\left\{\varphi_{t}: t \in \mathbb{R}\right\} \subset \operatorname{Aut}(G)
$$

must be a subgroup of $A u t(G)$, the Lie group of the automorphism of $G$. We call $\mathcal{X}$ an infinitesimal automorphism. Therefore, the drift is determined by the formula:

$$
\mathcal{X}(g)=\left(\frac{d}{d t}\right)_{t=o} \varphi_{t}(g)
$$


The Lie bracket between a linear and an invariant dynamic on $\mathbb{R}^{n}$ reads as:

$$
[A x, b]=-A b \in \mathbb{R}^{n} .
$$

Thus, the linear vector field determined by $A$ leaves invariant the Lie algebra $T_{0} \mathbb{R}^{n}=\mathbb{R}^{n}$ of $\mathbb{R}^{n}$. Therefore, we impose that $\mathcal{X}$ leaves invariant the Lie algebra $\mathfrak{g}$ of $G$ under the Lie brackets. In [19], the authors introduced the following notion:

Definition 2. A vector field $\mathcal{X}$ on $G$ is said to be linear if:

$$
[\mathcal{X}, Y] \in \mathfrak{g} \text {, for any } Y \in \mathfrak{g} \text {, and } \mathcal{X}(e)=0 .
$$

In [25], the author proved the following:

Theorem 4. Let $\mathcal{X}$ be a linear vector field on $G$. The following are equivalent:

1. $\mathcal{X}$ is linear;

2. $\mathcal{X}$ is an infinitesimal automorphism.

On the other hand, any vector $b \in \mathbb{R}^{n}$ determines by translation an invariant vector field $X^{b}$ on $\mathbb{R}^{n}$, as follows:

$$
X^{b}(x)=\sum_{i=0}^{n} b_{i}\left(\frac{\partial}{\partial x_{i}}\right)_{x} \in T_{x} \mathbb{R}^{n}
$$

Since,

$$
B u=\Sigma_{j=1}^{m} u_{j} b^{j}
$$

the linear control system $\Sigma_{\mathbb{R}^{n}}$ on $\mathbb{R}^{n}$ controls the matrix $A$ through $m$ invariant vector fields $X^{b_{1}}, \ldots, X^{b_{m}}$.

Let $G$ be a connected Lie group. In [19], the authors introduced the class of linear control systems $\Sigma_{G}$ on $G$ through the controlled family of differential equations:

$$
\Sigma_{G}: \dot{g}(t)=\mathcal{X}(g(t))+\sum_{j=1}^{m} u_{j}(t) X^{j}(g(t)), u \in \mathcal{U}
$$

parametrized as $u \in \mathcal{U}$. Here, the drift $\mathcal{X}$ is linear, $X^{1}, \ldots, X^{m}$ are invariant vector fields, and $u=\left(u_{1}, \ldots, u_{m}\right)$ belongs to the class of admissible control functions $\mathcal{U}=L_{\text {loc }}^{1}(\mathbb{R}, \Omega)$.

If $G=\mathbb{R}^{n}$, we recover the definition of the linear control system in Euclidean spaces. In other words, Formula (2) is a perfect generalization of Formula (1).

Remark 2. Any linear vector field $\mathcal{X}$ determines a derivation $\mathcal{D}=\mathcal{D}_{\mathcal{X}}$ of $\mathfrak{g}$, defined as:

$$
\mathcal{D}: \mathfrak{g} \rightarrow \mathfrak{g} \text { such that } \mathcal{D} X=-[\mathcal{X}, X](e), \text { for all } X \in \mathfrak{g} .
$$

Recall that $[A x, b]=-A b$ as in the Euclidean case. As a matter of fact, for each $t \in \mathbb{R},\left(d \varphi_{t}\right)_{e}=\mathrm{e}^{t \mathcal{D}}$. In particular, from the commutative diagram, we obtain:

$$
\begin{aligned}
& \begin{array}{ccc}
\mathfrak{g} & \stackrel{\left(d \varphi_{t}\right)_{e}}{\longrightarrow} & \mathfrak{g} \\
\exp \downarrow & & \downarrow \exp
\end{array} \underset{\varphi_{t}}{G} \quad \begin{array}{l}
G
\end{array} \\
& \varphi_{t}(\exp X)=\exp \left(d \varphi_{t}\right)_{e} X=\exp \left(\mathrm{e}^{t \mathcal{D}} X\right), \text { for all } t \in \mathbb{R}, X \in \mathfrak{g} \text {. }
\end{aligned}
$$


Furthermore, assume $G$ is also a simply connected and nilpotent Lie group. It is well known that in this case, the exponential map is a diffeomorphism. Consider a derivation $\mathcal{D}$, and for $g \in G$, take $X \in \mathfrak{g}$ such that $\log (g)=X$. Therefore,

$$
\varphi_{t}(g)=\exp \left(\mathrm{e}^{t \mathcal{D}} \log (g)\right), \text { for all } t \in \mathbb{R}, g \in G .
$$

Finally, consider the inner derivations $\mathcal{D} \in \partial \mathfrak{g}$, induced by an element $Y$ of $\mathfrak{g}$ by the formula $\mathcal{D}(\cdot)=[\cdot, Y]$. Since it flows:

$$
\varphi_{t}(g)=\exp (t Y) g \exp (-t Y), \text { for all } t \in \mathbb{R}
$$

is given by conjugation, and it is easy to determine the value of $\mathcal{X}=\mathcal{X}^{\mathcal{D}}$ on $G$ through the formula:

$$
\mathcal{X}=\left(\frac{d}{d t}\right)_{t=0} \varphi_{t}
$$

\subsection{The Solution of a Linear Control System on Lie Groups}

Let $\Sigma_{G}$ be a linear control system on the connected Lie group $G$. For a control $u \in \mathcal{U}$, the solution of:

$$
\dot{g(t)}=\mathcal{X}(g(t))+\sum_{j=1}^{m} u_{j}(t) X^{j}(g(t))
$$

with arbitrary initial condition $x \in G$ is given by:

$$
\gamma_{t}(x)=\varphi_{t}(\beta(t, u) x),
$$

where $\beta(t)$ is a differentiable curve in $\mathfrak{g}$. Precisely, this curve satisfies the differential equation:[19]

$$
\beta(t, u)=\left(\varphi_{-t}\right) *\left(\sum_{j=1}^{m} u_{j}(t) X_{\mathcal{X}(\odot(t), u)}^{j}\right) .
$$

\subsection{Observability Properties}

In this section, we state the main ideas and results appearing in [28]. First, we introduce the notion of a linear control system with an observation map on a Lie group. Through the shape of the solution of $\Sigma_{G}$ given in the previous section, we are willing to show that as in the Euclidean space, it is possible to characterize the observability property without the use of the control vectors. Actually, the algebraic condition just depends on the drift vector field and the observation map. Therefore, we have here the first symmetric situation between both classes of systems.

In this section, we will not show the proof of the main results. In fact, the mathematics involved are hard and out of the scope of this article.

For any homomorphism $h: G \rightarrow H$ between Lie groups, the kernel, and the image of $h$ :

$$
\operatorname{Ker}(h)=\left\{x \in G: h(x)=e_{H}\right\} \text { and } \operatorname{Im}(h)=\{y \in H: \exists x \in G \text { with } h(x)=y\}
$$

are subgroups of $G$ and $H$, respectively. Here, $e_{H}$ denotes the identity of $H$.

Definition 3. Let $G$ be a connected Lie group. A linear control system with observation $\Sigma_{G}^{\mathcal{O}}$ on $G$ is given by the data contained in Formula (2) and the observation map $h: G \stackrel{\text { hom }}{\rightarrow} H$, where $H$ is a Lie group with $\operatorname{dim}(G) \leq \operatorname{dim}(H)$.

We start with the indistinguishable and observable notions of $\Sigma_{G}^{\mathcal{O}}$. 
Definition 4. Let $\Sigma_{G}^{\mathcal{O}}$ be a linear control system with observation on $G$ :

1. Two elements $g_{1}$ and $g_{2}$ are indistinguishable, denoted by $g_{1} I g_{2}$, if:

$$
\varphi_{t}\left(g_{1} g_{2}^{-1}\right) \in \operatorname{Ker}(h), \forall t \geq 0
$$

2. $\Sigma_{G}^{\mathcal{O}}$ is said to be observable from $g \in G$ if the equivalence class $I(g)$ is $\{g\}$.

3. $\Sigma_{G}^{O}$ is said to be observable if it is observable from $g \in G$, for every $g \in G$.

4. $\Sigma_{G}^{\mathcal{O}}$ is said to be locally observable from $g \in G$ if $\exists U(g)$, a neighborhood of $g$, with:

$$
I(g) \cap U(g)=\{g\} .
$$

5. $\Sigma_{G}^{\mathcal{O}}$ is said to be locally observable if it is locally observable from $g, g \in G$.

Remark 3. In the Euclidean case, the equivalence class of the origin $I(0)$ is a subspace of $\mathbb{R}^{n}$. This means $I(0)$ is trivial or at least contains a line. In the non-trivial case, the local notion above does not have meaning. However, in the group case, $I(e)$ could be discrete and even finite, here $e=e_{G}$. Thus, the locally observable notion has perfect meaning. This is a fundamental difference between both Lie groups $\mathbb{R}^{n}$ and $G$.

The formula $\varphi_{t}\left(g_{1} g_{2}^{-1}\right) \in \operatorname{Ker}(h), \forall t \geq 0$, associated with the indistinguishable notion comes from the following analysis. Recall that $\varphi_{t}$ is an infinitesimal automorphism and $h$ is a homomorphism. Therefore, given two state $g_{1}$ and $g_{2}$ and any control $u \in \mathcal{U}$, we have:

$$
\begin{gathered}
g_{1} I g_{2} \Leftrightarrow h\left(\varphi_{t}\left(\beta(t, u) g_{1}\right)=h\left(\varphi_{t}\left(\beta(t, u) g_{2}\right) \Leftrightarrow\right.\right. \\
h\left(\varphi_{t}\left(g_{1}\right)\right)=h\left(\varphi_{t}\left(g_{2}\right)\right) \Leftrightarrow h\left(\varphi_{t}\left(g_{1} g_{2}^{-1}\right)=e_{H} .\right.
\end{gathered}
$$

In particular, by taking $g_{2}=e$, we obtain:

$$
I=I(e)=\left\{g \in G: \varphi_{t}(g) \in \operatorname{Ker}(h), \forall t \geq 0\right\} .
$$

Furthermore, for any $g \in G, I(g)=I g$. In fact,

$$
g_{1} I g_{2} \Leftrightarrow g_{2} \in I g_{1} .
$$

In the sequel, we denote $\Sigma_{G}^{\mathcal{O}}$ also by $(G, \mathcal{X}, h, H)$.

Proposition 2. Consider the system $\Sigma_{G}^{\mathcal{O}}=(G, \mathcal{X}, h, H)$. Then [28]:

1. I is a topologically closed and normal subgroup of $G$;

2. $I=\left\{g \in G: \varphi_{t}(g) \in \operatorname{Ker}(h), \forall t \in \mathbb{R}\right\}$.

Coming back to the Euclidean case, certainly, any subspace of $\mathbb{R}^{n}$ is topologically closed. Since $\mathbb{R}^{n}$ is an Abelian group, any subgroup is normal, actually Abelian as well. On the other hand, Proposition 1 implies that we have a complete symmetry between the indistinguishable class of the identity element $e$ of $G$ and the neutral element $0 \in \mathbb{R}^{n}$,

$$
I=\left\{x \in \mathbb{R}^{n}: \varphi_{t}(x) \in \operatorname{Ker}(h), \forall t \in \mathbb{R}\right\}
$$

\subsection{Local Observability}

As we mentioned, a continuous Lie group could contain discrete subgroups. For instance, for any natural number $k$, the set:

$$
R(k)=\left\{z \in \mathbb{C}: z^{k}=1\right\}
$$


is a finite subgroup of the circle $S^{1}$. For example, $R(4)=\left\{\frac{\pi}{2}, \pi, \frac{3 \pi}{2}, 1\right\}$.

In the sequel, we show an algebraic version of Proposition 17. Let $\mathcal{I}$ and $\mathcal{K}$ be the Lie algebras (the tangent space at the identity element $e$ ) of $I$ and the kernel $K=\operatorname{Ker}(h)$ of $h$, respectively. Define $a d^{i}(\mathcal{X}): \mathfrak{g} \rightarrow \mathfrak{g}$ inductively:

$$
a d^{0}(\mathcal{X})=I d, a d^{1}(\mathcal{X})(Y)=[\mathcal{X}, Y], \ldots, a d^{i}(\mathcal{X})=a d^{i-1}(\mathcal{X})([\mathcal{X}, Y]) .
$$

Proposition 3. Consider the system $\Sigma_{G}^{\mathcal{O}}=(G, \mathcal{X}, h, H)$. Then [28],

1. $a d^{i}(\mathcal{X})(\mathcal{I}) \subset \mathcal{I}$, for any natural number $i \geq 0$;

2. $\mathcal{I}=\cap_{i=0}^{n-1} a d^{-i}(\mathcal{X})(\mathcal{K})$.

The first condition means that the Lie bracket of any order between the drift $\mathcal{X}$ with elements in $\mathcal{I}$ will remain in $\mathcal{I}$. Precisely, let $Y \in \mathcal{I}$, then:

$$
[\mathcal{X}, Y] \in \mathcal{I},[\mathcal{X},[\mathcal{X}, Y]] \in \mathcal{I},[\mathcal{X},[\mathcal{X},[\mathcal{X}, Y]]] \in \mathcal{I}, \ldots \text { and so on. }
$$

The idea of the proof of 2 is as follows. Since $\mathcal{I} \subset \mathcal{K}$, we need to prove:

$$
\cap_{i=0}^{n-1} a d^{-i}(\mathcal{X})(\mathcal{K}) \subset \mathcal{K},
$$

which follows from 1.

Now, we are in a condition to state the main results about the observable system from the local point of view.

Theorem 5. Consider the system $\Sigma_{G}^{\mathcal{O}}=(G, \mathcal{X}, h, H)$. Then [28],

$$
\Sigma_{\mathbb{G}}^{\mathcal{O}} \text { is local observable } \Leftrightarrow \Sigma_{\mathbb{G}}^{\mathcal{O}} \text { is local observable from e } \Leftrightarrow \mathcal{I}=\{0\} .
$$

This result says that the observability property of $\Sigma_{\mathbb{R}^{n}}^{\mathcal{O}}$ on $\mathbb{R}^{n}$ and the local observability property of $\Sigma_{G}^{\mathcal{O}}$ on $G$ can be characterized in a symmetric way, i. e., with analogous mathematical tools. In fact,

$$
\mathcal{I}=\{0\} \text { is equivalent to } I=\{e\} .
$$

In fact, in the Euclidean case $e=0$. Therefore, we recover Theorem 3,

$$
\Sigma_{\mathbb{R}^{n}}^{\mathcal{O}} \text { is observable } \Leftrightarrow I=\{0\} .
$$

\subsection{Observability}

The local observability property of $\Sigma_{G}^{\mathcal{O}}$ just depends on the indistinguishable equivalence class of $e$. To obtain an observability result, we need to consider something more: the set of fixed points of the drift vector field $\mathcal{X}$, given by:

$$
\operatorname{Fix}(\mathcal{X})=\left\{g \in G: \varphi_{t}(g)=e, \text { for any } t \in \mathbb{R}\right\} .
$$

Theorem 6. Consider the system $\Sigma_{G}^{\mathcal{O}}=(G, \mathcal{X}, h, H)$. Then [28],

$$
\Sigma_{G}^{\mathcal{O}} \text { is observable } \Leftrightarrow \mathcal{I}=\{0\} \text { and } \operatorname{Fix}(\mathcal{X}) \cap \operatorname{Ker}(h)=\{e\} .
$$

Proof. Idea of the proof: Since $I=\{e\}$, we get $\mathcal{I}=\{0\}$. If $g \in \operatorname{Fix}(\mathcal{X}) \cap \operatorname{Ker}(h)$,

$$
\varphi_{t}(g)=g \in \operatorname{Ker}(h), \forall t \in \mathbb{R} .
$$


By definition, $g \in I$, then $g=e$.

Reciprocally, consider the map:

$$
\Phi: \mathbb{R} \times G \rightarrow G \text { defined by } \Phi(t, g)=\varphi_{t}(g) .
$$

We observe that $\Phi: \mathbb{R} \times I \rightarrow I$ is well defined. Pick $g \in G$, and define the application $\Phi_{g}: \mathbb{R} \times\{g\} \rightarrow G$. The set:

$$
\Phi_{g}(\mathbb{R} \times\{g\})=\operatorname{Im}\left(\Phi_{g}\right)=\left\{\varphi_{t}(g): t \in \mathbb{R}\right\}
$$

is connected since the domain $\mathbb{R} \times\{g\}$ is connected and $\Phi_{g}$ is continuous. By taking $t=0$, we get $g \in$ $\operatorname{Im}\left(\Phi_{g}\right) \subset I$.

$\mathcal{I}=\{0\}$ implies that $I$ is a discrete subgroup of $G$. Therefore,

$$
\{g\}=\operatorname{Im}\left(\Phi_{g}\right) \text { so } g \in \operatorname{Fix}(\mathcal{X}) .
$$

However, $g \in I \subset \operatorname{Ker}(h)$. It turns out that $g=e$, ending the proof.

\subsection{An Observability Algorithm}

Let us denote by $\mathfrak{g}^{\perp}$ the dual space of $\mathfrak{g}$. In order to compute the Lie algebra $\mathcal{I}$, it is suitable to use a general algorithm provided by Isidori [2]. Starting from the Lie algebra $\mathcal{K}$, we build a finite sequence of invariant $\mathfrak{g}^{\perp}$-subspaces, which converges to $\mathcal{I}^{\perp}$; see [28] for details. Let $\Sigma_{G}^{\mathcal{O}}=(G, \mathcal{X}, h, H)$ be a linear control system with observation. Consider the following steps:

1. Choose a basis $\mathcal{B}=\left\{Y^{1}, \ldots, Y^{p}\right\}$ of $\mathcal{K}$;

2. Compute the subspace $\mathcal{B}^{\perp}=\left\{w_{1}, \ldots, w_{n-p}\right\}$ of $\mathfrak{g}^{\perp}$;

3. Compute the $\operatorname{ad}(\mathcal{X})\left(\mathcal{B}^{\perp}\right)$-basis to $\mathcal{I}^{\perp}$, i.e.,

$$
\begin{gathered}
a d(\mathcal{X})\left(\mathcal{B}^{\perp}\right)=\left\{a d^{i}(\mathcal{X})\left(w_{j}\right) \mid 0 \leq i, 1 \leq j \leq n-p\right\}, \\
a d^{0}(\mathcal{X})=\operatorname{Id}, a d(\mathcal{X})(w)=w \circ a d(\mathcal{X}), \\
a d^{i}(\mathcal{X})(w)=a d\left(a d^{i-1}(\mathcal{X})(w)\right), i \geq 2 .
\end{gathered}
$$

The previous algorithm allows computing the relevant algebraic tool $\mathcal{I}$.

Theorem 7. Let us consider the system $\Sigma_{G}^{\mathcal{O}}=(G, \mathcal{X}, h, H)$. Therefore, [28]

$$
\mathcal{I}=\left(\operatorname{Spanad}(\mathcal{X})\left(\mathcal{B}^{\perp}\right)\right)^{\perp}
$$

Proof. It follows from Isidori's theorem in [2]. In fact, in this particular case, we obtain:

$$
\operatorname{Span}\left(\operatorname{ad}(\mathcal{X})\left(\mathcal{B}^{\perp}\right)\right)=\mathcal{I}^{\perp} .
$$

Recall that $I$ can be computed by integration of the Lie algebra $\mathcal{I}$.

\subsection{Examples}

In the sequel, we use the previous algorithm to compute some examples. 
Example 3. The Heisenberg-Lie group G of dimension three reads as:

$$
G=\left\{g=\left(\begin{array}{ccc}
1 & a & c \\
0 & 1 & b \\
0 & 0 & 1
\end{array}\right): a, b, c \in \mathbb{R}\right\}
$$

Let us denote by $e_{i j}^{*}$ the coefficient of a matrix such that the only non-null element is $e_{i j}=1$. The Lie algebra $\mathfrak{g}$ of $G$ is:

$$
\mathfrak{g}=\operatorname{Span}\left\{X^{1}=\left(e_{12}^{*}\right), X^{2}=\left(e_{23}^{*}\right), X^{3}=\left(e_{13}^{*}\right)\right\},
$$

where the only non-null bracket is $\left[X^{1}, X^{2}\right]=X^{3}$. We consider the infinitesimal automorphism $\mathcal{X}$ induced by the matrix $X^{1}$ by conjugation:

$$
\mathcal{X}(x)=\left(\frac{d}{d t}\right)_{t=0} \exp \left(t X^{1}\right) g \exp \left(-t X^{1}\right)=b X^{3}
$$

With these data, we develop two examples:

1. Let $\Sigma_{G}^{\mathcal{O}}=\left(G, \mathcal{X}, h=\pi_{2}, \mathbb{R}\right)$ be a linear control system on $G$, where:

$$
\pi_{2}\left(\begin{array}{lll}
1 & a & c \\
0 & 1 & b \\
0 & 0 & 1
\end{array}\right)=b
$$

We have,

$$
\operatorname{Ker}(h)=\left\{\left(\begin{array}{lll}
1 & a & c \\
0 & 1 & 0 \\
0 & 0 & 1
\end{array}\right): a, c \in \mathbb{R}\right\} \text { and } \mathcal{K}=\operatorname{Span}\left\{X^{1}, X^{3}\right\}
$$

Thus, the $\beta$ basis is $\left\{X^{1}, X^{3}\right\}$. It follows that the orthogonal basis $\beta^{\perp}$ is generated by $w=\left(X^{2}\right)^{\perp}$. Simple calculus shows that:

$$
\left[\mathcal{X}, X^{1}\right]=0,\left[\mathcal{X}, X^{2}\right]=X^{3} \text { and }\left[\mathcal{X}, X^{3}\right]=0
$$

Since $\operatorname{ad}(\mathcal{X})(w)=w \circ \operatorname{ad}(\mathcal{X})$, we obtain:

$$
\operatorname{ad}(\mathcal{X})(w)\left(X^{1}\right)=0, a d(\mathcal{X})(w)\left(X^{2}\right)=\left(X^{2}\right)^{\perp}\left(X^{3}\right)=0 \text { and } a d(\mathcal{X})(w)\left(X^{3}\right)=0 .
$$

Thus, $\operatorname{ad}(\mathcal{X})(w)=0$ and $a d^{i}(\mathcal{X})(w)$ for any $i$. It follows that:

$$
\{0\}=a d^{i}(\mathcal{X})\left(\beta^{\perp}\right) \subset \mathcal{K} \Rightarrow \mathcal{I}=\mathcal{K} .
$$

Consequently, $\Sigma_{G}^{\mathcal{O}}$ is not observable since it is not locally observable.

2. Let $\Sigma_{G}^{\mathcal{O}}=\left(G, \mathcal{X}, h, G / \exp \left(t X^{1}\right)\right.$ be a linear control system on $G$, where:

$$
h: G \rightarrow G / \exp \left(t X^{1}\right) \text { is the canonical projection. }
$$

We have,

$$
\operatorname{Ker}(h)=\left\{\left(\begin{array}{lll}
1 & 0 & 0 \\
0 & 1 & b \\
0 & 0 & 1
\end{array}\right): b \in \mathbb{R}\right\} \text { and } \mathcal{K}=\operatorname{Span}\left\{X^{2}\right\}
$$


Therefore, $\beta=\left\{X^{2}\right\}$ and $\beta^{\perp}=\left\{w_{1}=\left(X^{1}\right)^{\perp}, w_{2}=\left(X^{3}\right)^{\perp}\right\}$. However, $a d(\mathcal{X})(w)=\left(X^{2}\right)^{\perp}$. Then, $\mathcal{I}^{\mathcal{I}}=\mathfrak{g}^{\mathcal{I}}$ and $\mathcal{I}=\{0\}$, which implies by Theorem 19 that the system $\Sigma_{G}^{\mathcal{O}}$ is locally observable.

On the other hand, the set of fixed points on the drift:

$$
\begin{aligned}
& \operatorname{Fix}(\mathcal{X})=\left\{\left(\begin{array}{lll}
1 & a & c \\
0 & 1 & 0 \\
0 & 0 & 1
\end{array}\right): a, c \in \mathbb{R}\right\}, \text { in fact } \\
& \varphi_{t}(g)=\exp \left(t X^{1}\right) g \exp \left(-t X^{1}\right)=g \Leftrightarrow b=0 .
\end{aligned}
$$

It turns out that the only element in $\operatorname{Fix}(\mathcal{X})$ and $\operatorname{Ker}(h)$ is the identity. From Theorem 6, the system $\Sigma_{G}^{\mathcal{O}}=\left(G, \mathcal{X}, h, G / \exp \left(t X^{1}\right)\right.$ is observable.

\section{Conclusions}

In this paper, we first showed that the symmetric property of the Gramian matrix determines totally the observability property of any linear control system on a Euclidean space.

Second, the natural extension of a linear control system from $\mathbb{R}^{n}$ to a Lie group $G$ contains many symmetries. In fact, the drift $A$ of $\Sigma_{\mathbb{R}^{n}}$ with flow $e^{t A} \in A u t\left(\mathbb{R}^{n}\right), t \in \mathbb{R}$, can be generalized to the drift $\mathcal{X}$ of $\Sigma_{G}$ through the infinitesimal automorphism notion. Precisely, its flow $\varphi_{t} \in A u t(G)$, for any $t \in \mathbb{R}$. Furthermore, the column vectors of the matrix $B$ of $\Sigma_{\mathbb{R}^{n}}$ determine $m$ constant vector fields on $\mathbb{R}^{n}$, which can be generalized as $m$ invariant vector fields on the Lie group $G$.

On the other hand, we proved that there are many symmetries in the given data, which allow characterizing the observability properties of $\Sigma_{\mathbb{R}^{n}}^{\mathcal{O}}$ and $\Sigma_{G}^{\mathcal{O}}$. For instance,

1. The equivalent class $I(0)$ of $0 \in \mathbb{R}^{n}$ and $I(e)$ of $e \in G$ have symmetric constructions;

2. The algebraic tool $I$ allows characterizing the observability property of $\Sigma_{\mathbb{R}^{n}}^{\mathcal{O}}$. The natural extension of $I=I(0)$ from a subspace to a subgroup $I=I(e)$ characterizes the locally observable property of $\Sigma_{G}^{\mathcal{O}}$.

It is worth saying that contrary to the Abelian group $\mathbb{R}^{n}$, a Lie group $G$ could contain discrete subgroups. To approach this relevant difference, we considered the sets of fixed points of the drift vector fields $\mathcal{X}$. With that, we characterized the observability of $\Sigma_{G}^{\mathcal{O}}$.

Author Contributions: Supervision, V.A.; writing, original draft, M.T.T. and H.R.-F.; writing, review and editing, E.Z. All authors have read and agreed to the published version of the manuscript.

Funding: Se agradece a la Universidad Nacional de San Agustín de Arequipa, UNSA, por el financiamiento del proyecto de investigación según Contrato $\mathrm{N}^{\circ}$ IAI-014-2018-UNSA.

Conflicts of Interest: The authors declare no conflict of interest.

\section{Appendix A. A Short Review of Lie Theory}

To define the class of linear control systems in a more general setting, we introduce some basic notion of Lie theory; mainly, matrix Lie groups, Lie algebras, and two special classes of dynamics: invariant and linear vector fields. For general facts of this matter, we mention the following references $[6,7,29,32]$.

A group $G$ is said to be a topological group if the applications $\mu: G \times G \rightarrow G$, defined by $\mu(g, h)=g h$ and $\iota: G \rightarrow G$, determined by $\iota(g)=g^{-1}$ are continuous.

According to the solved Hilbert's fifth problem, $G$ has a structure of differential manifolds, and $\mu$ and $\iota$ are analytical applications. We call this structure a Lie group. In particular, it is possible to define the differential equation on $G$ (vector fields) and to compute its solution through its flows. 
Example A1. The following sets are Lie groups with the natural structure:

1. The Euclidean space $\left(\mathbb{R}^{n},+\right)$.

2. The multiplicative set $G L(n, \mathbb{R})=\{A: \operatorname{det}(A) \neq 0\}$ of invertible real matrices of order $n$. We denote by $\mathrm{GL}^{+}(n, \mathbb{R})$ the connected component, which contains the identity map Id.

3. The $n$-dimensional torus $T^{n}=S^{1} \times \ldots \times S^{1}$, n-times, where $S^{1}=\{z \in \mathbb{C}:|z|=1\}$ is the circle group.

4. The special linear group:

$$
S L(n, \mathbb{R})={ }^{-1} \operatorname{det}(1)
$$

and the special orthogonal group:

$$
S O(n, \mathbb{R})=\left\{A \in O(d) \mid A A^{t}=I d \text { and } \operatorname{det}(A)=1\right\}
$$

5. A matrix representation of the Heisenberg-Lie group reads as:

$$
G=\left\{g=\left(\begin{array}{ccc}
1 & a & c \\
0 & 1 & b \\
0 & 0 & 1
\end{array}\right): a, b, c \in \mathbb{R}\right\} .
$$

Define the right and the left translations maps on $G$ by:

$$
R_{g}: G \rightarrow G, R_{g}(x)=x g \text { and } L_{g}: G \rightarrow G, L_{g}(x)=g x
$$

respectively. Their inverses are given by $R_{g^{-1}}$ and $L_{g^{-1}}$.

Definition A1. An invariant vector field $X$ on $G$ is determined by the formula $X_{g}=\left(R_{g}\right)_{*}\left(X_{e}\right), g \in G$.

Here, $\left(R_{g}\right)_{*}$ denotes de derivative of $R_{g}$ at the identity element $e$ of $G$. Just observe that any invariant vector field is determined by its value at $e$.

The notion of Lie brackets is a measure of the non-commutative behavior between any two dynamics. In the case of a matrix group, the Lie bracket corresponds to the commutator, $[A, B]=A B-B A$.

Here is an example coming from real life. Imagine an airplane flying always at the same altitude and moving $1000 \mathrm{~km}$ in every direction: first to the north, second to the west, then to the south, and finally, to the east. Except for some special states, the initial and ending points of the trip are always different!The plane moves through the solution of two vector fields on the sphere $S^{2}=$ $\left\{x \in \mathbb{R}^{3}:\|x\|=1\right\}$ induced by rotational matrices $A, B \in S O(d, \mathbb{R})$. In this case, $[A, B] \neq 0$.

Denote by $\mathfrak{g}$ the vector space of right invariant vector fields on $G$. As a matter of fact,

$$
X, Y \in \mathfrak{g} \Rightarrow[X, Y] \in \mathfrak{g} .
$$

Therefore, $\mathfrak{g}$ is isomorphic to the tangent space $T_{e} G$, and it is called the Lie algebra $\mathfrak{g}$ of $G$. The elements of $\mathfrak{g}$ satisfy the skew-symmetric property:

$$
[X, Y]=-[Y, X], \text { for any } X, Y \in \mathfrak{g}
$$

and the Jacobi identity:

$$
\operatorname{ad}(X)([Y, Z])+\operatorname{ad}(Z)([X, Y])+a d(Y)([Z, X])=0, \text { if } X, Y, Z \in \mathfrak{g}
$$

Moreover, any subspace $V \subset \mathfrak{g}$ is a subalgebra if:

$$
X, Y \in V \Rightarrow[X, Y] \in V
$$


$V$ is an ideal if:

$$
X \in V \text { and } Y \in \mathfrak{g} \Rightarrow[X, Y] \in V .
$$

Next, we show the algebra $\mathfrak{g}$ of some groups through $T_{e} G$ :

1. $\quad T_{0} \mathbb{R}^{n}=\mathbb{R}^{n}$;

2. $\quad T_{I d} G L^{+}(n, \mathbb{R})=\mathfrak{g l}(n, \mathbb{R})$;

3. $T_{e} S^{2}=\mathbb{R}^{2}$;

4. $\quad T_{I d} S O(n, \mathbb{R})=\left\{A \in \mathfrak{g l}(n, \mathbb{R}) \mid A+A^{t}=0\right\}$, the skew-symmetric matrices;

5. $\quad \mathfrak{s l}(n, \mathbb{R})=\{A \in \mathfrak{g l}(n, \mathbb{R}) \mid \operatorname{tr}(A)=0\}$, the trace zero matrices;

6. The Lie algebra of the Heisenberg group has the basis $\left\{X^{1}, X^{2}, X^{3}\right\}$ such that $\left[X^{1}, X^{2}\right]=X^{3}$.

The exponential map exp $: \mathfrak{g} \rightarrow G$ defined on any invariant vector field $X \in \mathfrak{g}$ reads as:

$$
\exp (X)=X_{t}(e)_{t=1}=X_{1}(e)
$$

In the classical case of the matrix group, we recover:

$$
\exp : \mathfrak{g l}(d, \mathbb{R}) \rightarrow G L^{+}(d, \mathbb{R}), \exp A=\left(e^{t A}\right)_{(t=1)}=e^{A} .
$$

Since $d(\exp )_{0}=e, \exists V \subset G$ a neighborhood of $e$ where exp is a diffeomorphism (local). This fact comes from the inverse map theorem [32].

The set $\operatorname{Aut}(G)$ of $G$-automorphisms is a Lie group with Lie algebra aut $(G)$ when $G$ is connected [32-35]. Furthermore, if $G$ is also simply connected, aut $(G)$ is isomorphic to the Lie algebra $\partial \mathfrak{g}$ of $\mathfrak{g}$-derivations, i.e., the set of linear maps $D: \mathfrak{g} \rightarrow \mathfrak{g}$, which satisfy the Leibniz rule $D[X, Y]=[D X, Y]+[X, D Y]$.

A remarkable relationship between a homomorphism and its derivative is given by the commutative diagram:

$$
\begin{array}{lcl}
\mathfrak{g} & \text { The derivative at e } & \mathfrak{h} \\
\exp _{\mathfrak{g}} \downarrow & \stackrel{\overrightarrow{d(\varphi)_{e}}}{\hookrightarrow} & \downarrow \exp _{\mathfrak{h}} \\
G & \underset{\varphi}{\stackrel{\hookrightarrow}{\longrightarrow}} & H
\end{array}
$$

As an example, we recover the very well-known formula involving the determinant and the trace of a matrix:

$$
e^{\operatorname{tr} A}=\operatorname{det}(\exp A), A \in \mathfrak{g l}(d, \mathbb{R}) .
$$

where $G=G L^{+}(n, \mathbb{R}), \mathfrak{g}=\mathfrak{g l}(\mathfrak{n}, \mathbb{R}), H=\mathbb{R}$, and $\mathfrak{h}=\mathbb{R}$.

Definition A2. A Lie algebra $\mathfrak{g}$ is said to be:

1. Abelian if every bracket is null;

2. Nilpotent if the sequence $\operatorname{ad}^{j}(\mathfrak{g})=\left[\operatorname{ad}^{j-1}(\mathfrak{g}), \operatorname{ad}^{1}(\mathfrak{g})\right], j=1,2, \ldots$ stabilizes at zero, i.e.,

$$
\exists k \geq 1: \operatorname{ad}^{k}(\mathfrak{g})=\left[\operatorname{ad}^{k-1}(\mathfrak{g}), \operatorname{ad}^{1}(\mathfrak{g})\right]=0
$$

3. Solvable if the sequence $\operatorname{ad}^{(j)}(\mathfrak{g})=\left[\operatorname{ad}^{(j-1)}(\mathfrak{g}), \operatorname{ad}^{(j-1)}(\mathfrak{g})\right] j=1,2, \ldots$ stabilizes at zero, i.e.,

$$
\exists k \geq 1: \operatorname{ad}^{(k)}(\mathfrak{g})=\left[\operatorname{ad}^{(k-1)}(\mathfrak{g}), \operatorname{ad}^{(k-1)}(\mathfrak{g})\right]=0
$$

4. Simple if $\mathfrak{g}$ is not Abelian and does not contain non-proper ideals;

5. Semisimple if the solvable radical $\mathfrak{r}(\mathfrak{g}$ ) (the largest solvable Lie subalgebra of $\mathfrak{g}$ ) is null. 
We classify a Lie group with the same name that is inherited from the classification of its Lie algebra.

\section{Example A2.}

1. $\mathbb{R}^{n}$ is Abelian;

2. $T^{m}=S^{1} \times \ldots \times S^{1}$ is Abelian and compact;

3. $\mathbb{R}^{n} \times T^{n}, n, m \in \mathbb{N}$ is the general Abelian group shape;

4. The three-dimensional Heisenberg group is nilpotent;

5. The affine group $\left\{\left(\begin{array}{cc}A & y \\ 0 & 1\end{array}\right): A \in G L(n, \mathbb{R}), y \in \mathbb{R}^{n}\right\}$ is solvable;

6. $S O(n, \mathbb{R})$ is compact:

$$
S O(n, \mathbb{R})=\left\{A \in G L(n, \mathbb{R}) \mid A A^{t}=e\right\}
$$

7. $S O(4, \mathbb{R})$ is compact and semisimple;

8. $S L(n, \mathbb{R})$ is non-bounded and semisimple.

\section{References}

1. Colonius, F.; Kliemann, W. The Dynamics of Control; Birkhäuser: Boston, MA, USA, 2000.

2. Isidori, A. Nonlinear Control Systems; Springer: Cham, Switzerland 1998. [CrossRef]

3. Jurdjevic, V. Geometric Control Theory; Cambridge University Press: Cambridge, UK, 1997. ISBN 0521058244; ISBN 9780521058247.

4. Pontryagin, L.S.; Boltyanskii, V.G.; Gamkrelidze, R.V.; Mishchenko, E.F. The Mathematical Theory of Optimal Processes; English translation; Interscience: Hoboken, New Jersey,1962. [CrossRef]

5. Sachkov, Y. Control Theory on Lie Groups; Lecture Notes SISSA: Trieste, Italy, 2006. [CrossRef]

6. Helgason, S. Differential Geometry, Lie Groups and Symmetric Spaces; Academic Press: New York, NY, USA, 1978.

7. San Martin, L.A.B. Algebras de Lie; Editorial UNICAMP: Campinas, Brazil, 1999.

8. Varadarajan, V. Lie Groups, Lie Algebras and their Representations; Prentice Hall, Inc.: Upper Saddle River, NJ, USA, 1974. [CrossRef]

9. Axelby, G.S. Round-Table Discussion on the Relevance of Control Theory. Automatica 1973, 9, 279-281. [CrossRef]

10. Lee, D.H.; Milroy, I.P.; Tyler, K. Application of Pontryagin's Maximum Principle to the Semi-automatic Control of Rail Vehicles. In Proceedings of the Second Conference on Control Engineering 1982: Merging of Technology and Theory to Solve Industrial Automation Problems, Newcastle, Australia, 25-27 August 1982; pp. 233-236.

11. Leitmann, G. Optimization Techniques with Application to Aerospace Systems; Academic Press Inc.: London, UK, 1962.

12. Markus, L. Controllability of Multi-Trajectories on Lie Groups. Lect. N.a. Math. 1980. [CrossRef]

13. Pesch, H.J.; Plail, M. The Cold War and the Maximum Principle of Optimal Control; Documenta Mathematica; Extra Volume ISMP; 2012, pp. 331-343. Avaliable online: https:/ / www.math.uni-bielefeld.de/documenta/ vol-ismp/48_pesch-hans-josef-cold-war.pdf (accessed on 1 January 2012).

14. Shell, K. Applications of Pontryagin's Maximum Principle to Economics. In Mathematical Systems Theory and Economics I and II, Volume 11/12; The Series Lecture Notes in Operations Research and Mathematical Economics; Springer: Berlin/Heidelberg, Germany, 1969; pp. 241-292.

15. Naidu, D.S. Optimal Control Systems; CRC Press: Washington D.C., USA, 2002.ISBN:0-8493-0892-5.

16. Ledzewicz, U.; Shattler, H. Optimal Control for a Bilinear Model with Recruiting Agent in Cancer Chemotherapy. In Proceedings of the MTNS, Kyoto, Japan, 24-28 July 2006.

17. Ledzewick, U.; Shattler, H. Optimal controls for a two compartment model for cancer chemotherapy with quadratic objective. In Proceedings of the MTNS, Kyoto, Japan, 24-28 July 2006.

18. Sussmann, H.; Willems, C. 300 Years of Optimal Control: From the Brachystochrone to the Maximum Principle. IEEE Control. Hist. Perspect. 1997, 17, 32-44. [CrossRef]

19. Ayala, V.; Tirao, J. Linear Control Systems on Lie Groups and Controllability. AMS Proc. Symp. Pure Math. 1999, 64, 47-64. 
20. Ayala, V.; Da Silva, A. On the characterization of the controllability property for linear control systems on nonnilpotent, solvable three-dimensional Lie groups. J. Differ. Equ. 2019, 266, 8233-8257. [CrossRef]

21. Ayala, V.; Da Silva, A. The control set of a linear control system on the two dimensional Lie group. J. Differ. Equ. 2020, 268, 6683-6701. [CrossRef]

22. Ayala, V.; Da Silva, A.; Jouan, P.; Zsigmond, G. Control sets of linear systems on semi-simple Lie groups. J. Differ. Equ. 2020, 269, 449-466. [CrossRef]

23. Ayala, V.; Da Silva, A. A Semigroup associated to a linear control system on a Lie group. Syst. Control. Lett. 2016, 98, 33-36. [CrossRef]

24. Ayala, V.; Jouan, P. Almost Riemannian Geometry on Lie Groups. Siam J. Control Optim. 2016, 54, $2919-2947$. [CrossRef]

25. Jouan, P. Equivalence of Control Systems with Linear Systems on Lie Groups and Homogeneous Spaces. ESAIM Control Optim. Calc. Var. 2010, 16, 956-973 [CrossRef]

26. Jouan, P. Controllability of Linear Systems on Lie groups. J. Dyn. Control Syst. 2011, 17, 591-616. [CrossRef]

27. Ayala, V.; Kizil, E.; Hacibekiroglu, A. Observability of General Linear Pairs. Comput. Math. Appl. 2001, 39, 35-43. [CrossRef]

28. Ayala, V.; Hacibekiroglu, A.K. Observable Linear Pairs. Comput. Appl. Math. 1997, 16, $205-214$.

29. Curtis, L.M. Matrix Groups; Springer: New York, NY, USA, 1984. doi:10.1007/978-1-4612-5286-3

30. Wonham, M.W. Linear Multivariable Control: A Geometric Approach; Applications of Mathematics; Springer: New York, NY, USA, 1979; Volume 10, p. 326. ISBN 978-1-4612-1082-5

31. Kalman, R. Lecture Notes on Controllability and Observability; Springer International Publishing: Cham, Switzerland, 1968; ISBN 978-3-642-11063-4.

32. Warner, F.W. Foundations of Differential Manifolds and Lie Groups; Scott Foreman: Glenview, IL, USA, 1971.

33. Ayala, V.; San Martín, L. Controllability Properties of a Class of Control Systems on Lie Groups; Lectures Notes in Control and Information Science; Springer, London, UK, 2001. [CrossRef]

34. Ayala, V.; Da Silva, A. Controllability of Linear Control Systems on Lie Groups with Semisimple Finite Center. SIAM J. Control. Optim. 2017, 55, 1332-1343. [CrossRef]

35. Kalman, R.; Ho, Y.; Narendra, K. Controllability of Linear Dynamical Systems. Contrib. Diff. Equ. 1962, 1, 189-213.

(C) 2020 by the authors. Licensee MDPI, Basel, Switzerland. This article is an open access article distributed under the terms and conditions of the Creative Commons Attribution (CC BY) license (http://creativecommons.org/licenses/by/4.0/). 